\title{
Identificação das espécies brasileiras de Akodon (Rodentia: Cricetidae: Sigmodontinae) através da microestrutura dos pelos
}

\author{
Fabiana Silveira $^{1,4}$, Ives José Sbalqueiro² \& Emygdio Leite de Araujo Monteiro-Filho ${ }^{1,3}$ \\ ${ }^{1}$ Laboratório de Biologia e Ecologia de Vertebrados, Departamento de Zoologia, \\ Universidade Federal do Paraná - UFPR, CP 19020, CEP 81531-980, Curitiba, PR, Brasil \\ ${ }^{2}$ Laboratório de Citogenética, Departamento de Genética, Universidade Federal do Paraná - UFPR, \\ CP 19071, CEP 81531-980, Curitiba, PR, Brasil \\ ${ }^{3}$ Instituto de Pesquisas Cananéia - IPeC, Rua Tristão Lobo, 199, Centro, \\ CEP 11990-000, Cananéia, SP, Brasil \\ ${ }^{4}$ Autor para correspondência: Fabiana Silveira, e-mail: fabianasilveira@ufpr.br
}

SILVEIRA, F., SBALQUEIRO, I.J. \& MONTEIRO-FILHO, E.L.A. Identification of the brazilian species of Akodon (Rodentia: Cricetidae: Sigmodontinae) through the microstructure of the hair. Biota Neotrop. 13(1): http://www.biotaneotropica.org.br/v13n1/en/abstract?article+bn00713012013

\begin{abstract}
There are 10 species of Akodon Meyen, 1833 in Brazil and most have some degree of geographic overlap, even with records of sympatry between some species. The identification of the species is difficult and can be performed by the analysis of the morphological structure of hair. Thus, in order to identify the microstructure of the nine Brazilian species of Akodon, guard-hairs samples from zoological collections were used. We adopted the method of analysis of the cuticle scales and medulla. The cuticle pattern for all species was narrow leaf shaped. For the medulla three basic patterns have been recognized: alveolar multiseriate, multiseriate striped and a mixture of alveolar and striped. Akodon cursor is the only species which has a predominance of four layers of cells, the specimens $2 \mathrm{n}=14$ and $2 \mathrm{n}=15$ have an alveolar medulla and the specimen $2 \mathrm{n}=16$, has a striped medulla and elongated cells. For Akodon paranaensis and A. lindberghi the medulla alternates between three and four layers. The others species have three layers. Akodon mystax, have a larger spaces between the cells. Akodon reigi shows an intermediary sector with layers of oval and well connected cells and for Akodon toba, the cells of the central layer are found in one or two small alveolar layers. In the striped pattern, Akodon montensis have narrow intercellular space in regard to the thickness of the cell. In a mixed pattern, Akodon azarae presents cells with clear outline, and Akodon serrensis show cells with irregular shape. Thus, the use of the microstructure of hairs as a tool for identification of the species of Akodon proved to be practicable.
\end{abstract}

Keywords: rodents, cuticle scales, guard hair, morphologie, medulla.

SILVEIRA, F., SBALQUEIRO, I.J. \& MONTEIRO-FILHO, E.L.A. Identificação das espécies brasileiras de Akodon (Rodentia: Cricetidae: Sigmodontinae) através da microestrutura dos pelos. Biota Neotrop. 13(1): http://www.biotaneotropica.org.br/v13n1/pt/abstract?article+bn00713012013

Resumo: No Brasil há 10 espécies de Akodon Meyen, 1833 e a maioria apresenta algum grau de sobreposição geográfica havendo inclusive registros de simpatia. A identificação das espécies é difícil e pode ser feita pela análise da estrutura morfológica de pelos. Assim, para a identificação da microestrutura de pelos de nove espécies brasileiras de Akodon, foram utilizados pelos-guardas primários de amostras de coleções zoológicas. Foi adotado o método de análise das escamas cuticulares e da medula. O padrão de cutícula para todas as espécies foi folidáceo estreito. Já para a medula foram reconhecidos três padrões básicos como multisseriada alveolar, multisseriada listrada e misto de alveolar e listrada. Akodon cursor é a única espécie com predomínio de quatro fileiras sendo que exemplares $2 n=14$ e $2 n=15$ apresentam medula alveolar e o $2 n=16$, medula listrada e células longilíneas. Para Akodon paranaensis e A. lindberghi a medula se alterna entre três e quatro fileiras. As demais espécies apresentam três fileiras. Akodon mystax apresenta um maior espaçamento entre as células. Akodon reigi possui o setor intermediário com fileiras ovaladas e bem ligadas e Akodon toba tem as células da fileira central variando com uma e duas células alveolares pequenas. No padrão listrado, Akodon montensis apresenta espaço intercelular mais estreito em relação à espessura da célula. No padrão misto, Akodon azarae apresenta células com contorno evidente, já Akodon serrensis as células apresentam formato irregular. Assim, o uso da microestrutura dos pelos como ferramenta para a identificação das espécies de Akodon mostrou-se perfeitamente viável.

Palavras-chave: roedores, escamas cuticulares, pelos-guarda, morfologia, medula. 


\section{Introdução}

Os roedores pertencentes ao gênero Akodon Meyen, 1833, são distribuídos por toda a América do Sul sendo representados por 41 espécies (Musser \& Carleton 2005), cujos estudos na sua grande maioria, são de caráter sistemático e desenvolvidos na Argentina (Del Valle \& Busch 2003, Pereira et al. 2005, Pardiñas \& Teta 2007, Braun et al., 2008, 2010, Pardiñas 2009), no Brasil (Fagundes et al. 1998, Geise et al. 1998, Sbalqueiro \& Nascimento 1996, Fagundes \& Nogueira 2007, Queirolo \& Granzinolli 2009), na Bolívia (Myers \& Patton 1989a, Braun et al., 2008), no Chile (Simonetti et al. 1985, Meserve et al. 1991, Antinuchi \& Busch 2000), no Paraguai (Yahnke 2006, Braun et al., 2008) e, no Peru e Bolívia (Myers \& Patton 1989b, Myers et al. 1990).

Particularmente para o Brasil, são consideradas 10 espécies (Paglia et al. 2012) e a exceção de A. toba (Thomas, 1921), todas as espécies brasileiras apresentam algum grau de sobreposição em suas áreas de distribuição (Bonvicino et al. 2008), havendo inclusive registros de simpatria (Fagundes et al. 1998, Geise et al. 1998) como o que ocorre entre Akodon cursor (Winge, 1887) e Akodon montensis (Thomas, 1913) nos estados do Rio de Janeiro, de Minas Gerais, de São Paulo e norte do Paraná (Fagundes \& Nogueira 2007) e, entre A. lindberghi (Hershkovitz, 1990) e A. cursor no Estado de Minas Gerais (Geise et al. 1998).

A diferenciação dessas espécies é feita com base em aspectos morfológicos incluindo a análise de crânios (Geise et al. 2005, Pardiñas 2009, Braun et al., 2010) assim como com a utilização de técnicas genéticas, entre elas as análises moleculares (Geise et al. 1998, Silva et al. 2006, Queirolo \& Granzinolli 2009, Braun et al., 2010). Entretanto, essas técnicas requerem investimentos maiores e se tornam inviáveis na identificação individual em estudos de ecologia de populações (Fagundes \& Nogueira 2007).

Toda esta dificuldade na identificação é comum para a maioria dos roedores e deve ser considerada em estudos de cunho ecológico onde é fundamental a marcação e soltura dos animais (Lessa et al. 1999, Feliciano et al. 2002, Graipel et al. 2006), nos estudos de dieta de carnívoros em que os roedores são frequentemente consumidos (Bernarde et al. 2000, Magrini 2006, Martins et al. 2008, Rinaldi 2010) e até mesmo na identificação de espécimes em coleções científicas na ausência dos crânios, ocasiões onde é fundamental a utilização de técnicas rotineiras como, por exemplo, a identificação de espécies através da estrutura morfológica de pelos. Esta técnica é amplamente utilizada em diferentes partes do mundo (Keller 1986, Wallis 1992, Vanek \& Keller 1993, Ingberman \& MonteiroFilho 2006, Juárez et al. 2009, entre outros) e permite a utilização independente do pelo ser oriundo de animais vivos, ter passado por diferentes tratamentos como os processos de decomposição, banho ácido no trato gastrintestinal ou até mesmo processos básicos de taxidermia em coleções científicas (Quadros \& Monteiro-Filho 1998).

Assim, considerando a grande dificuldade em diferenciar morfologicamente as espécies de Akodon, somada à possibilidade de ocorrência de algumas espécies em simpatria e de variações cromossômicas existentes dentro da mesma espécie (Fagundes \& Nogueira 2007), nosso intuito é identificar nove espécies brasileiras do gênero Akodon com base na microestrutura do pelo e avaliar se há alguma característica morfológica do pelo que possa estar relacionada com a conhecida variação cromossômica existente para a espécie Akodon cursor.

\section{Material e Métodos}

As amostras de pelos foram coletadas de animais taxidermizados e devidamente identificados de Akodon que ocorrem no Brasil, os quais são provenientes dos acervos do Museu Nacional, Universidade
Federal do Rio de Janeiro (MNRJ, Rio de Janeiro, RJ), Laboratório de Citogenética da Universidade Federal do Paraná (LCUFPR, Curitiba, PR) e acervo do professor Doutor Nilton C. Cáceres da Universidade Federal de Santa Maria (UFSM, URUCUM, JST, GE, JSE) (Tabela 1). As amostras recebidas são de exemplares coletados em diferentes localidades evitando assim a utilização de características que pudessem representar potenciais variações geográficas. Para algumas espécies há um número menor de amostras e isso se deve ao pequeno número de animais tombados nos acervos. Não foi possível obter amostras de Akodon sanctipaulensis (Hershkovitz, 1990).

A coleta de pelos seguiu o método proposto por Quadros \& Monteiro-Filho (2006a) que consiste em retirar com os dedos, em torno de dez pelos da parte dorsal entre as escapulas dos espécimes. Os pelos foram acondicionados em envelopes de papel e identificados com nome da espécie, dados da coleção de origem e informação citogenética, quando existente.

Os pelos foram então lavados com álcool a 70\% e sob uma lupa foram escolhidos somente os pelos-guardas primários, os quais foram posteriormente secos em papel absorvente. Para as impressões das escamas cuticulares foi espalhada uma fina camada de esmalte incolor em uma lâmina, deixado secar por cerca de 30 minutos, depois os pelos foram colocados sobre a fina camada de esmalte e prensados. Buscando a secagem do esmalte e uma melhor impressão da cutícula, os pelos foram retirados após 12 horas e examinados em sua haste (que é a parte proximal do pelo em relação à epiderme, delgada e varia entre reta e ondulada, cf. Quadros \& Monteiro-Filho 2006b). Após a secagem, os pelos foram retirados com os dedos e imersos em água oxigenada cremosa comercial 30 volumes por 80 minutos com o propósito de diafanizá-los para a análise morfológica da medula no escudo (parte mais alargada e situa-se entre a haste e a extremidade distal do pelo, cf. Quadros \& Monteiro-Filho 2006b). Estes pelos foram então lavados e depois montados entre lâmina e lamínula tendo como meio de montagem Entelan. Para obter um melhor resultado na diafanização, alguns pelos foram lavados em água com detergente para a retirada de resíduos de gordura, que impediria a perfeita diafanização. Para a diagnose correta foi necessária a utilização de no mínimo cinco pelos de cada amostra. Para a visualização das impressões cuticulares e da medula foi utilizado microscópio óptico nos aumentos de $100 \times$ e $400 \times$.

Devido à ocorrência de variação do número cromossômico para a espécie $A$. cursor, três exemplares com $2 \mathrm{n}=14$, sete exemplares com $2 \mathrm{n}=15$ e quatro exemplares com $2 \mathrm{n}=16$ foram analisados visando a testar se estas variações cromossômicas poderiam ser detectadas também na morfologia dos pelos.

Para a descrição da medula e cutícula foi utilizado os padrões descritos por Quadros \& Monteiro-Filho (2006b).

\section{Resultados}

Foram analisadas 54 amostras de nove espécies brasileiras do gênero Akodon e o número de amostras variou de acordo com a disponibilidade de exemplares nas coleções. Para A. mystax (Hershkovitz 1998), A. toba (Thomas 1921), A. reigi (González et al. 1998), A. lindberghi (Hershkovitz 1990) foram obtidas duas amostras de cada, A. azarae (Fischer 1829) quatro amostras, A. paranaensis (Christoff et al. 2000) sete amostras, A. serrensis (Thomas, 1902) nove amostras, A. montensis (Thomas 1913), dez amostras e A. cursor (Winge 1887) 16 amostras.

O padrão de cutícula registrado para as nove espécies brasileiras estudadas do gênero Akodon foi folidáceo estreito (Figura 1). De uma maneira geral, a morfologia da medula também é bastante parecida, mas permite o reconhecimento de três padrões básicos 
Tabela 1. Espécies analisadas, local de coleta das amostras e acervos de depósito.

Table 1. Analyzed species, location of sample and collection of reference.

\begin{tabular}{|c|c|c|}
\hline Espécie & Local de coleta & Acervo de depósito \\
\hline \multirow[t]{2}{*}{ Akodon azarae } & Taim, Rio Grande, RS & JSTII, JST20 \\
\hline & Espinilho, Barra do Quarai, RS & JSE 17, JSE170 \\
\hline \multirow[t]{9}{*}{ Akodon cursor } & Ilha do Rabelo, Guaraqueçaba, PR. & LCUFPR 636, 784 \\
\hline & Ilha das Laranjeiras, Guaraqueçaba, PR. & LCUFPR 666, 748 \\
\hline & Índios, Guaraqueçaba, PR. & LCUFPR 758,762 \\
\hline & Ilha Rasa, Guaraqueçaba, PR. & LCUFPR 681 \\
\hline & Ilha das Gamelas, Guaraqueçaba, PR & LCUFPR 736 \\
\hline & Massarapuã, Guaraqueçaba, PR. & LCUFPR 742, 749, 744, 751 \\
\hline & Tromomô, Guaraqueçaba, PR & LCUFPR 745, 771 \\
\hline & Bom Conselho, PE & MNRJ 19117 \\
\hline & Garanhuns, PE & MNRJ 19118 \\
\hline Akodon lindberghi & Simão Pereira, MG & MNRJ 33686, 33687 \\
\hline \multirow[t]{9}{*}{ Akodon montensis } & Morretes, PR & LCUFPR 795 \\
\hline & Roça Nova, Piraquara, PR & LCUFPR 878 \\
\hline & Botuquara, Palmeira, PR & LCUFPR 989 \\
\hline & São Luís do Purunã, PR & LCUFPR 1001 \\
\hline & Barro Preto, São José dos Pinhais, PR & LCUFPR 1012 \\
\hline & Itatiaia, RJ & MNRJ 48031 \\
\hline & Nova Friburgo, RJ & MNRJ 55733 \\
\hline & Turvo, Derrubada, RS & GE51, GE97 \\
\hline & Morro do Elefante, Santa Maria, RS & UFSM 527 \\
\hline Akodon mystax & Parque Nacional do Caparaó, MG & MNRJ 33686, 33687 \\
\hline \multirow[t]{4}{*}{ Akodon paranaensis } & Curitiba, PR & LCUFPR 153 \\
\hline & ADEA, São José dos Pinhais, PR & LCUFPR 884 \\
\hline & Barro Preto, São José dos Pinhais, PR & LCUFPR 983, 987, 1024 \\
\hline & Itatiaia, RJ & MNRJ: 69685,69686 \\
\hline Akodon reigi & Taim, Rio Grande, RS & JST3, JST6 \\
\hline \multirow[t]{3}{*}{ Akodon serrensis } & Quatro Barras, PR & LCUFPR 828,831,833,834,835 \\
\hline & Piraquara, $\mathrm{PR}$ & LCUFPR 854,888 \\
\hline & Parque Nacional do Caparaó, MG & MNRJ: 69621,69640 \\
\hline \multirow[t]{2}{*}{ Akodon toba } & Santa Cruz/ Urucum, Corumbá, MS & UFSM 258 \\
\hline & Morro Santa Cruz, Corumbá, MS & URUCUM 58 \\
\hline
\end{tabular}

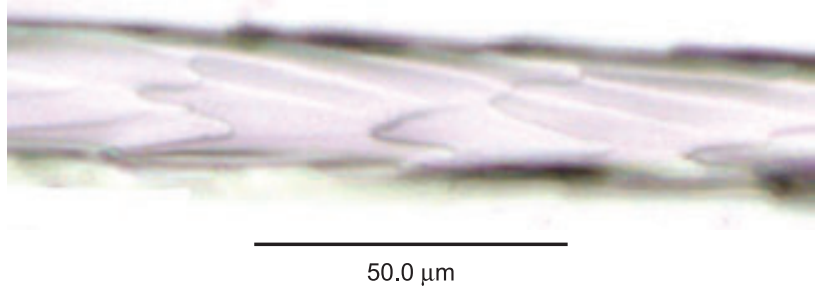

Figura 1. Padrão cuticular folidáceo estreito observado nos exemplares do gênero Akodon que ocorrem no Brasil (aumento de $400 \times$ ).

Figure 1. Cuticular narrow leaf shaped of the genus Akodon occurring in Brazil (amplified 400×).

como multisseriada alveolar, multisseriada listrada e um misto entre alveolar e listrada (Figura 2).

Foi possível detectar variação na microestrutura do pelo dentro das variações cromossômicas de $A$. cursor. Para as variações $2 n=14$ e 15 foi obtido um padrão alveolar, já para a variação $2 \mathrm{n}=16$ ocorreu o padrão listrado. Foi constatado que nas amostras de Akodon cursor ocorre a presença de muito sub-pelo que é ondulado, curto e com constrições, os quais não devem ser utilizados por serem semelhantes entre amostras de espécies diferentes (cf. Quadros \& MonteiroFilho 2006b). Para evitar confundir o sub-pelo com o pelo-guarda é recomendado utilizar pelos com escudo bem largos.
Akodon cursor é a única espécie que apresenta predomínio de quatro fileiras. Para Akodon paranaensis, A. lindberghi, A. azarae e $A$. serrensis a medula se alterna entre três e quatro fileiras. Akodon mystax se diferencia das demais espécies devido ao maior espaçamento entre as células. Akodon reigi apresenta o setor intermediário com fileiras ovaladas e bem ligadas e Akodon toba as células da fileira central variando com uma e duas células alveolares pequenas. Akodon montensis se distingue das demais por apresentar espaço intercelular mais estreito em relação à espessura da célula (Tabela 2). Para visualizar o padrão misto é necessário olhar o pelo em panorâmica (aumento de $100 \times$ ) e posteriormente utilizar o maior aumento para diferenciar A. azarae de A. serrensis.

\section{Discussão}

O uso da microestrutura dos pelos é um método de baixo custo, de fácil acesso e rápido que pode ser utilizado em estudos que enfocam a relação predador/presa (Quadros \& Monteiro-Filho 2010), além dos estudos forenses (Sato et al. 2009) e levantamento de mastofauna (Martin et al. 2009) entre tantos outros.

Alguns estudos já descreveram a microestrutura de pelos de algumas espécies de Akodon que não ocorrem no Brasil como para A. iniscatus, A. longipilis, A. olivaceus, A. sanborni, A. xanthorhinus (Chehébar \& Martín 1989) e para Akodon molina (Fernández \& Rossi 1998). Nestes estudos a cutícula dos pelos apresenta o mesmo padrão 
Silveira, F. et al.

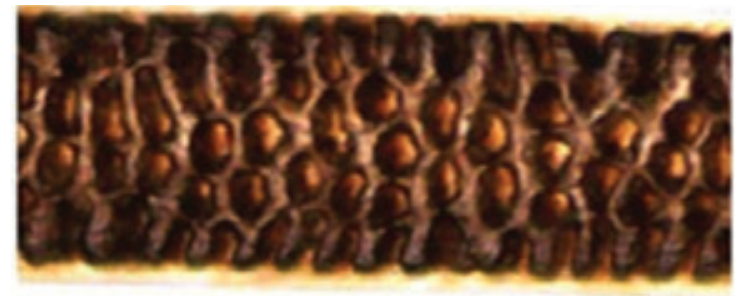

$50.0 \mu \mathrm{m}$

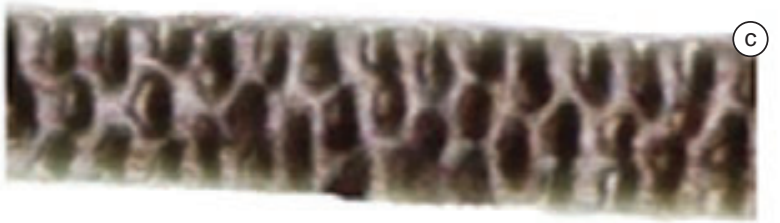

$50.0 \mu \mathrm{m}$

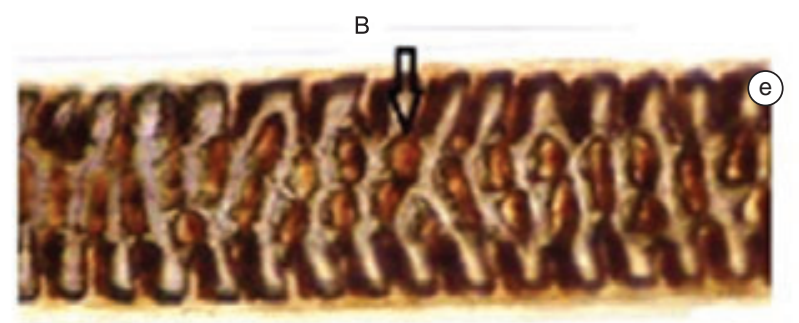

$50.0 \mu \mathrm{m}$

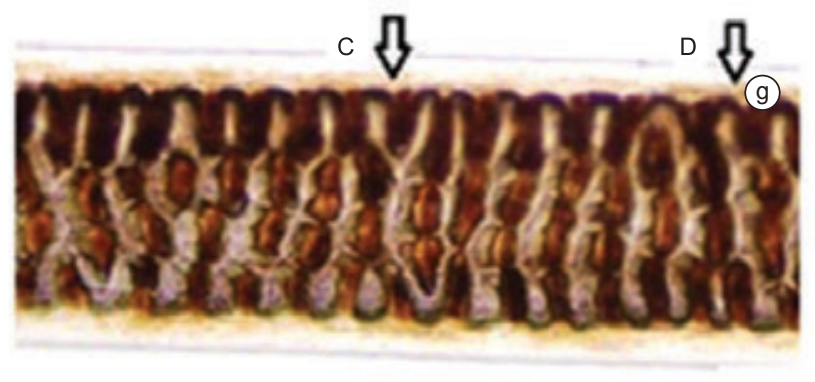

$50.0 \mu \mathrm{m}$

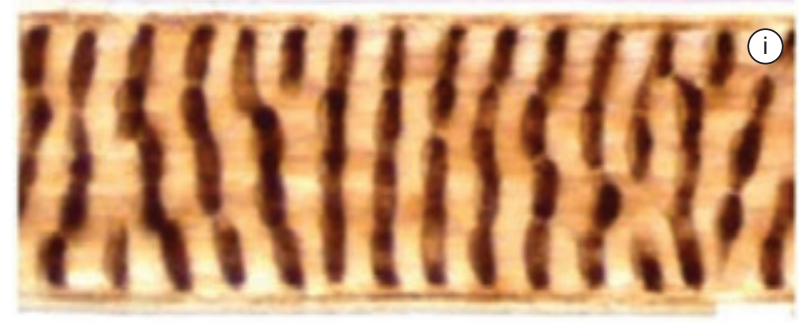

$50.0 \mu \mathrm{m}$

(a)

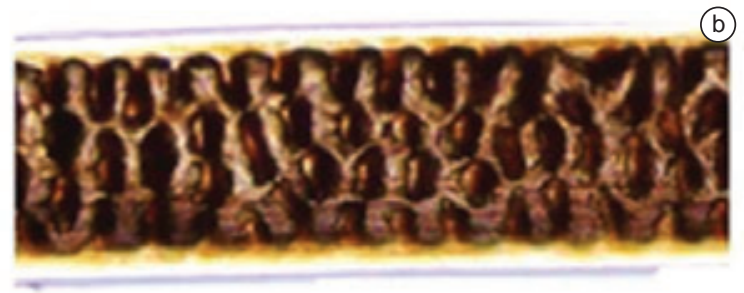

$50.0 \mu \mathrm{m}$

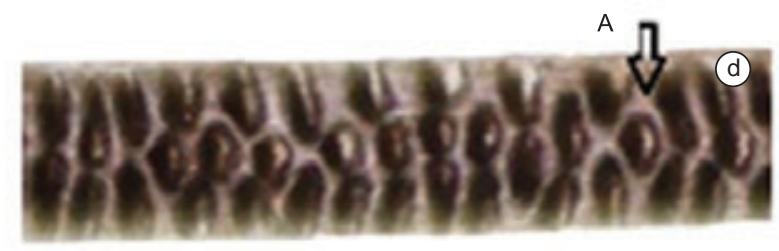

$50.0 \mu \mathrm{m}$

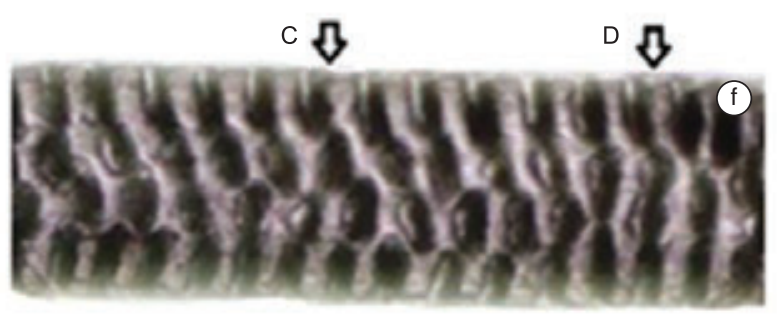

$50.0 \mu \mathrm{m}$

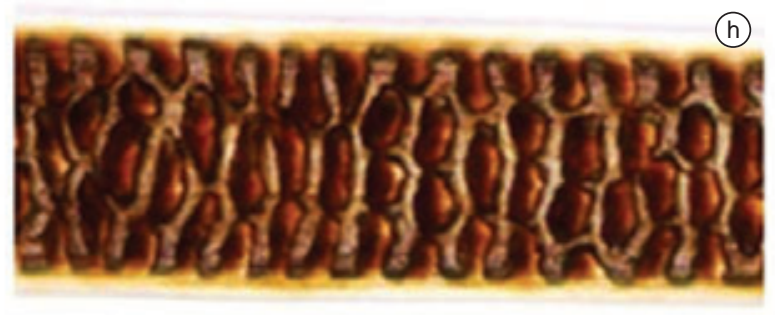

$50.0 \mu \mathrm{m}$

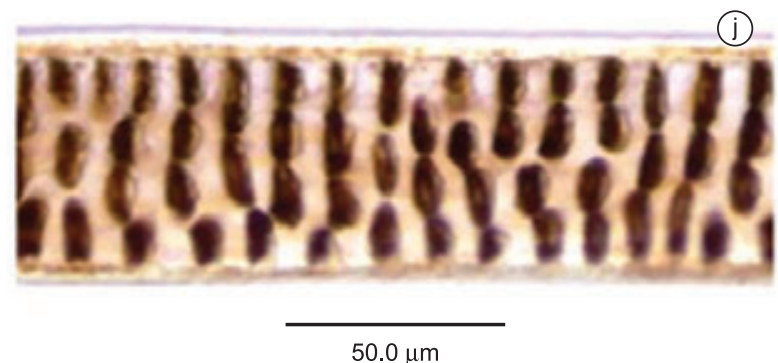

Figura 2. Características microestruturais diagnósticas da medula para a identificação dos pelos-guarda das espécies de $A k o d o n$ que ocorrem no Brasil (aumento de 400×). a) Akodon cursor ( $2 \mathrm{n}=14$ e 15), b) Akodon paranaensis, c) Akodon mystax, d) Akodon reigi, seta A indica as células ovaladas, e) Akodon toba, seta $\mathrm{B}$ indica as cálulas alveolares pequenas, f) Akodon azarae, seta C indica padrão alveolar, seta $\mathrm{D}$ indica padrão listrado, g) Akodon serrensis, seta C indica padrão alveolar, seta D indica padrão listrado, h) Akodon montensis, i) Akdon cursor $(2 \mathrm{n}=16)$ e j) Akodon lindberghi.

Figure 2. Microstructural characteristics of medulla of the hair-guard of Akodon species of the Brazil (amplified 400 $\times$ ). a) Akodon cursor (2n = 14 and 15), b) Akodon paranaensis, c) Akodon mystax, d) Akodon reigi, arrow A indicates oval cells, e) Akodon toba, arrow B indicates the alveolar small cells, f) Akodon azarae, arrow C indicates alveolar pattern, arrow D indicates striped pattern, g) Akodon serrensis, arrow C indicates alveolar pattern, arrow D indicates striped pattern, h) Akodon montensis, i) Akdon cursor $(2 \mathrm{n}=16)$ and j) Akodon lindberghi. 
Tabela 2. Chave dicotômica de identificação das espécies brasileiras do gênero Akodon através da microestrutura dos pelos.

Table 2. Dichotomous key for the identification of the brazilian species of Akodon through the microstructure of the hair.

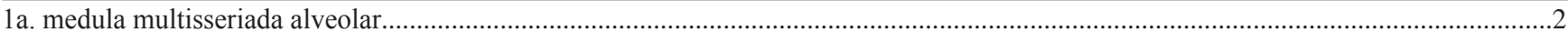

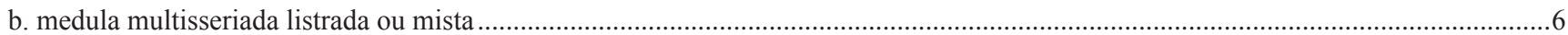

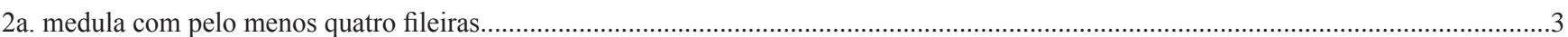

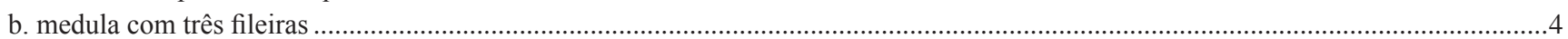

3a. medula com predomínio de quatro fileiras, podendo ocorrer cinco fileiras................................Akodon cursor ( $2 \mathrm{n}=14 \mathrm{e} 15$; Figura $2 \mathrm{a})$

b. medula alternando entre três e quatro fileiras .......................................................................................Akodon paranaensis (Figura 2b)

4a. fileira central com células distribuídas de forma irregular...........................................................................Akodon mystax (Figura 2c)

b. fileira central com células distribuídas em sequências uniformes ...........................................................................................5

5a. setor intermediário do escudo com pelo menos três fileiras bem ovaladas e ligadas ........................................ Akodon reigi (Figura 2 d)

b. fileira central variando com uma e duas células alveolares pequenas ..............................................................Akodon toba (Figura 2e)

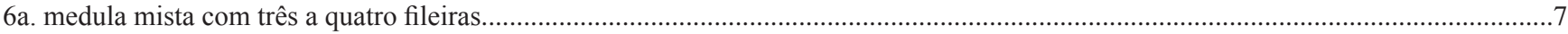

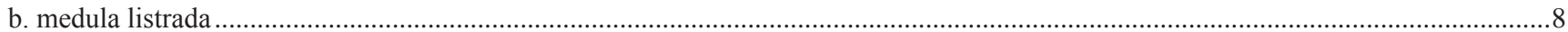

7a. células com contorno definido ...........................................................................................................kodon azarae (Figura 2f)

b. células com formato irregular .................................................................................................................. Akodon serrensis (Figura 2g)

8a. espaço intercelular estreito em relação a espessura da célula .....................................................................Akodon montensis (Figura 2h)

b. espaço intercelular grande em relação a espessura da célula...........................................................................................................9

9a. predomínio de quatro fileiras e células longilíneas ....................................................................Akodon cursor (2n = 16; Figura 2i)

b. variação entre três e quatro fileiras de células e células ovaladas............................................................Akodon lindberghi (Figura 2j)

existente para as espécies brasileiras mesmo considerando a utilização de outra nomenclatura e a medula, é predominantemente composta por três fileiras de células, assim como ocorre para a maioria dos padrões medulares agora descritos. Assim, é possível que o tipo de arranjo das escamas cuticulares existentes na haste dos pelos seja um padrão característico do gênero, mas o número de fileiras, a distribuição e o formato das células medulares do escudo parecem ser variáveis, permitindo assim o reconhecimento específico, pelo menos para as espécies estudadas.

Para as espécies que ocorrem no Brasil só existiam as descrições feitas para a estrutura dos pelos de A. cursor (Quadros 2002) e A. montensis (Martin et al. 2009). Entretanto, como estes estudos reconheceram uma espécie de Akodon além de outros gêneros, as diagnose utilizadas buscaram detalhar grandes grupos e, portanto, não utilizaram as mesmas características agora descritas para um conjunto de nove espécies morfologicamente muito semelhantes. Quando a descrição ocorre dentro de um gênero as características utilizadas precisam ser mais esmiuçadas como apresentada no nosso estudo. Assim, é esperado que as características diagnósticas adotadas para grandes grupos separados filogeneticamente e aquelas adotadas dentro de um mesmo gênero acabem sendo muito diferentes.

Para o desenvolvimento de estudos que utilizam os padrões medulares e cuticulares como ferramenta para o reconhecimento de espécies, são necessários estudos sistemáticos prévios. Contudo, algumas propostas de caráter sistemático divergem periodicamente na dependência do desenvolvimento e aplicação de novas técnicas. Este é o caso que ocorre com os espécimes nominados como $A$. cursor que de acordo com os estudos de Geise et al. (2001) e Silva et al. (2006), os com $2 n=16$ são entidades biológicas distintas das de $2 n=14$ e $2 n=15$, ou ainda, de acordo com Nogueira \& Fagundes (2008) e Barros et al. (2009) que consideram estes três cariótipos pertencentes a uma única espécie. Em nosso estudo, analisando exemplares de A. cursor com os três números de cariótipos, detectamos que como um todo, esta é a espécie com maior número de fileiras de células e facilmente identificada com base na estrutura da medula quando comparada aos demais representantes do gênero no Brasil, mas a despeito disto, dois padrões distintos de medula puderam ser reconhecidos, ou seja, um com medula alveolar $(2 n=14$ e $2 n=15)$ e o outro com medula listrada
$(2 \mathrm{n}=16)$. Neste caso, a variação encontrada na morfologia dos pelos de $A$. cursor, parece refletir a variação genética já documentada por Geise et al. (2001) e de Silva et al. (2006).

Assim, o uso dos pelos como ferramenta de identificação das espécies brasileiras de Akodon é perfeitamente viável permitindo sua utilização em estudos de ecologia e na identificação de animais inclusive os de museu.

\section{Agradecimentos}

Agradecemos a João Alves Oliveira do Museu Nacional do Rio de Janeiro e Nilton C. Cáceres da Universidade Federal de Santa Maria pelo envio das amostras. As fotos foram obtidas com o auxílio dos professores Thelma Ludwig (Equipamento obtido pelo processo 555397/2006-8 do Ed. MCT/ CNPq/CTHidro) e Fernando Sedor. A Karin D. K. A. Monteiro pela revisão do Abstract. Aos revisores anônimos desse periódico pelas valiosas considerações e sugestões. Os autores receberam patrocínio do CNPq (FS- Bolsa de mestrado; ELAMF- Bolsa de Produtividade de Pesquisa).

\section{Referências Bibliográficas}

ANTINUCHI, C.D. \& BUSCH, C. 2000. Metabolic rates and thermoregulatory characteristics of Akodon azarae (Rodentia: Sigmodontinae). Rev. Chil. Hist. Nat.. 73: 131-138. http://dx.doi.org/10.4067/S0716078X2000000100012

BARROS, M.C., SAMPAIO, I., SCHNEIDER, H. \& LANGGUTH, A. 2009.Molecular phylogenies, chromosomes and dispersion in Brazilian akodontinies (Rodentia, Sigmodontinae).Iheringia, Sér. Zool. 99(4):373-380.

BERNARDE, P.S., MOURA-LEITE, J.C., MACHADO, R.A. \& KOKOBUM, M.N. C. 2000. Diet of the colubrid snake, Thamnodynastes strigatus (Günther, 1858) from Parana State, Brazil, with field notes on anuran predation. Rev. Bras. Biol. 60(4):695-699. http://dx.doi.org/10.1590/ S0034-71082000000400022

BRAUN, J.K., COYNER, B.S., MARES, M.A. \&; VAN DEN BUSSCHE, R.A. 2008. Phylogenetic relationships of South American grass mice of the Akodon various group (Rodentia, Cricetidae, Sigmodontinae) in South America. J. Mammal. 89(3):768-777. http://dx.doi.org/10.1644/07MAMM-A-269R1.1 
BRAUN, J.K., MARES, M.A., COYNER, B.S. \& VAN DEN BUSSCHE, R.A. 2010. New species of Akodon (Rodentia: Cricetidae: Sigmodontinae) from central Argentina. J. Mammal. 91(2):387-400. http://dx.doi. org/10.1644/09-MAMM-A-048.1

BONVICINO, C.R., OLIVEIRA, J.A. \& D'ANDREA, P.S. 2008. Guia dos roedores do Brasil, com chaves para gêneros baseadas em caracteres externos. Centro Pan-Americano de Febre Aftosa - OPAS/OMS.

CHEHÉBAR, C. \& MARTÍN, S. 1989. Guía para ela reconocimiento microscópico de los pelos de los mamíferos de la Patagonia. Doñana, Acta Vertebr. 16(2):247-291.

DEL VALLE, J.C. \& BUSCH, C. 2003. Body composition and gut length of Akodon azarae (Muridae: Sigmodontinae): relationship with energetic requirements. Acta Theriol. 48(3):347-357. http://dx.doi.org/10.1007/ BF03194174

FAGUNDES, V., CHRISTOFF, A.U. \& YONENAGA-YASSUDA, Y. 1998. Extraordinary chromosomal polymorphism with 28 different karyotypes in the neotropical species Akodon cursor (Muridae, Sigmodontinae), one of the smallest diploid number in rodents ( $2 \mathrm{n}=16,15$ and 14$)$. eHereditas 129:263-274. http://dx.doi.org/10.1111/ j.1601-5223.1998.00263.x

FAGUNDES, V. \& NOGUEIRA, C.D.A. 2007. The use of PCR-RFLP as an identification tool for three closely related species of rodents of the genus Akodon (Sigmodontinae, Akodontini). Genet. Mol. Biol. 30(3):698-701.

FELICIANO, B.R., FERNANDEZ, F.A.S., FREITAS, D. \& FIGUEIREDO, S.L. 2002. Population dynamics of small rodents in a grassland between fragments of Atlantic Forest in southeastern Brazil. Mamm. Biol. 67:304-314. http://dx.doi.org/10.1078/1616-5047-00045

FERNÁNDEZ, G.J. \& ROSSI, S.M. 1998. Medullar type and cuticular scale patterns of hairs of rodents and small marsupials from the Monte Scrubland (San Luis province, Argentina). Mastozool. Neotrop. 5:109-116.

GEISE, L., CANAYEZ, F.C. \& SEUÁNEZ, H.N. 1998. Comparative Karyology in Akodon (Rodentia, Sigmodontinae) from Southeastern Brazil. J. Heredity. 89:158-163. http://dx.doi.org/10.1093/jhered/89.2.158

GEISE, L., SMITH, M.F. \& PATTON, J.L. 2001. Diversification in the genus Akodon (Rodentia, Sigmodontinae) in southeastern America: Mitochondrial DNA sequences analysis. J. Mammal. 82:92-101. http:// dx.doi.org/10.1644/1545-1542(2001)082<0092:DITGAR>2.0.CO;2

GEISE, L., MORAES, D.A. \& SILVA, H.S. 2005. Morphometric differentiation and distributional notes of three species of AKodon (Muridae, Sigmodontinae, Akodontini) in the Atlantic coastal area of Brazil. Arq. Mus. Nac.63 (1):63-74.

GRAIPEL, M.E., CHEREM, J.J., MONTEIRO-FILHO, E.L.A. \& GLOCK, L. 2006. Dinâmica populacional de marsupiais e roedores no Parque Municipal da Lagoa do Peri, Ilha de Santa Catarina, sul do Brasil. Mastozool. Neotrop. 13(1):31-49.

INGBERMAN, B. \& MONTEIRO-FILHO, E.L.A. 2006. Identificação microscópica dos pelos das espécies brasileiras de Alouatta Lacépéde, 1799 (Primates, Atelidae, Alouattinae). Arq. Mus. Nac.64(1):61-71.

JUÁREZ, D., ESTRADA, C., BUSTAMANTE, M., QUINTANA, Y., MOREIRA, J. \& LÓPEZ, J. 2009. Guía ilustrada de pelos para la identificación de mamíferos mayores y medianos de Guatemala. Universidad de San Carlos de Guatemala, Guatemala.

KELLER, A. 1986. Etude comparative de la structure fine des poils dês Pipistrelles d Europe (Mammalia: Chiroptera). Revue Suisse Zool. 93:409-415.

LESSA, G., GONÇALVES, P.R., MORAIS JÚNIOR, M.M., COSTA, F.M., PEREIRA, R.F. \& PAGLIA, A.P. 1999. Caracterização e monitoramento da fauna de pequenos mamíferos terrestres de um fragmento de mata secundária em Viçosa, Minas Gerais. Bios. 7(7):41-49.

MAGRINI, L. 2006. Predação de pequenos mamíferos por suindara (Tyto alba) e seu papel no controle de reservatórios naturais de hantavírus em uma periurbana do município de Uberlândia, Minas Gerais, Brasil. Dissertação de mestrado, Universidade Federal de Uberlândia, Minas Gerais.
MARTIN, P.S., GHELER-COSTA, C. \& VERDADE, L.M. 2009. Microestruturas de pelos de pequenos mamíferos não-voadores: chave para identificação de espécies de agroecossistemas do estado de São Paulo, Brasil. Biota Neotrop. 9(1): http://www.biotaneotropica.org.br/ v9n1/pt/abstract?identification-key+bn01509012009 (último acesso em 10/09/2011).

MARTINS, R., QUADROS, J. \& MAZZOLLI, M. 2008. Hábito alimentar e interferência antrópica na atividade de marcação territorial do Puma concolor e Leopardus pardalis (Carnivora: Felidae) e outros carnívoros na Estação Ecológica de Juréia-Itatins, São Paulo, Brasil. Rev. Bras. Zool. 25(3):427-435. http://dx.doi.org/10.1590/S010181752008000300007

MESERVE, P.L., LANG, B.K., MURUA, R., MUÑOZ-PEDREROS, A., GONZALEZ, L.A. 1991. Characteristics of a terrestrial small mammal assemblage in a temperate rainforest in Chile. Rev. Chil. Hist. Nat. 64:157-169.

MUSSER, G.G. \& CARLETON, M.D. 2005. Superfamily Muroidea. In Mammal species of the world. A taxonomic and geographic reference (D.E. Wilson \& D.M. Reeder, eds). The John Hopkins University Press, Baltimore, v.2, p.894-1531.

MYERS, P. \& PATTON, J.L. 1989a. A new species of Akodon from the cloud forests of eastern Cochabamba Department, Bolivia (Rodentia: Sigmodontinae). Occas. Pap. Mus. Zool. 720:1-28.

MYERS, P. \& PATTON, J.L. 1989b. Akodon of Peru and Bolivia- Revision of the fumeus group (Rodentia: Sigmodontinae). Occas. Pap. Mus. Zool. 721:1-35.

MYERS, P., PATTON, J.L. \& SMITH, M.F. 1990. A review of the Boliviensis group of Akodon (Muridae: Sigmodontinae), with emphasis on Peru and Bolivia. Miscellaneous Publications of the Museum of Zoology, University of Michigan, 177.

NOGUEIRA, C.A. \& FAGUNDES, V. 2008. Akodon cursor Winge, 1887 (Rodentia: Sigmodontinae): one or two species? New evidences based on molecular data. Zootaxa 1768:41-51.

PAGLIA, A.P., FONSECA, G.A.B., RYLANDS, A.B., HERMANN, G., AGUIAR, L.M.S., CHIARELLO, A.G., LEITE, Y.L.R., COSTA, L.P., SICILIANO, S., KIERULFF, M.C.M., MENDES, S.L., TAVARES, V. C., MITTERMEIER, R.A. \& PATTON, J.L. 2012. Lista Anotada dos Mamíferos do Brasil. 2. ed. Occasional papers in conservation Biology. Conservation Internacional, Arlington, V.A. 6.

PARDIÑAS, U.F.J. \& TETA, P. 2007. Micromamíferos del sector oriental de la antiplanicie del Somuncurá (Río Negro, Argentina). Mastozool. Neotrop. 14(2):271-278.

PARDIÑAS, U.F.J. 2009. El Género Akodon (Rodentia: Cricetidae) em Patagonia: Estado actual de seu conocimiento. Mastozool. Neotrop. 16(1):135-152.

PEREIRA, J., TETA, P., FRACASSI, N., JOHNSON, A. \& MOREYRA, P. 2005. Sigmodontinos (Rodentia, Cricetidae) de la Reserva de Vida Silvestre Urugua-Í (Provincia de Missiones, Argentina) com la confirmácion de la presença de "Akodon" serrensis em la Argentina. Mastozool. Neotrop. 12(1):83-89.

QUADROS, J. \& MONTEIRO-FILHO, E.L.A. 1998. Effects of digestion, putrefaction and taxidermy processes on Didelphis albiventris hair morphology. J. Zool. 244(4):331-334. http://dx.doi. org/10.1111/j.1469-7998.1998.tb00037.x

QUADROS, J. 2002. Identificação microscópica de pelos de mamíferos brasileiros e sua aplicação no estudo da dieta de carnívoros. Tese de doutorado, Universidade Federal do Paraná. Paraná.

QUADROS, J. \& MONTEIRO-FILHO, E.L.A. 2006a. Coleta e preparação de pelos de mamíferos para identificação em microscopia óptica. Rev. Bras. Zool. 23: 274-278. http://dx.doi.org/10.1590/S0101-81752006000100022

QUADROS, J. \& MONTEIRO-FILHO, E.L.A. 2006b. Revisão conceitual, padrões microestruturais e proposta nomenclatória para os pelos-guarda de mamíferos brasileiros. Rev. Bras. Zool. 3:279-292. http://dx.doi. org/10.1590/S0101-81752006000100023 
QUADROS, J. \& MONTEIRO-FILHO, E.L.A. 2010. Identificação dos mamíferos de uma área de floresta Atlântica utilizando a microestrutura de pelos-guarda de predador e presa. Arq. Mus. Nac.68(1-2):47-66.

QUEIROLO, D. \& GRANZINOLLI, M.A.M. 2009. Ecology and natural history of Akodon lindberghi (Rodentia, Sigmodontinae) in southeastern Brazil. Iheringia, Sér. Zool. 99(2):189-193.

RINALDI, A.R. 2010. Dieta de pequenos felinos silvestres (Carnívora, Felidae), em área antropizada de Mata Atlântica de Interior, Alto Paraná, Paraná, Brasil. Dissertação de mestrado, Universidade Federal do Paraná, Paraná.

SATO, I., NAKAKI, S., MURATA, K., TAKESHITA, H. \& MUKAI, T. 2009. Forensic hair analysis to identify animal species on a case of pet animal abuse. Int J Legal Med. 124: 249-256. http://dx.doi.org/10.1007/s00414009-0383-2

SBALQUEIRO, I.J. \& NASCIMENTO, A.P. 1996. Occurrence of Akodon cursor (Rodentia, Cricetidae) with 14, 15 and 16 chromosome cytotypes in the same geographic area in Southern Brazil. Rev. Bras. Genet. 19:565569. http://dx.doi.org/10.1590/S0100-84551996000400005
SILVA, M.J., PATTON, J.L. \& YONENAGA-YASSUDA, Y. 2006. Phylogenetic relationships and karyotype evolution in the sigmodontine rodent Akodon ( $2 \mathrm{n}=10$ and $2 \mathrm{n}=16$ ) from Brazil. Genet. Mol. Biol. 29(3):469-474. http://dx.doi.org/10.1590/S1415-47572006000300012

SIMONETTI, J.A., FUENTES, E.R. \& OTAIZA, R.D. 1985. Habitat use by two rodent species in the high Andes of central Chile. Mammalia. 49(1):19-25. http://dx.doi.org/10.1515/mamm.1985.49.1.19

VANEK, M. \& KELLER, A. 1993. Etude de la morphologie dês écailles de la cuticule dês jarres primaires dês renards polaires adultes - Alopex lagopus (Linné, 1758) à 1 aide Du microscope électronique à balayage. Revue Suisse Zool. 100(4):899-903.

WALLIS, R.L. 1992. A key for the identification of guard hairs of some Ontario mammals. Can. J. Zool. 71:587-591. http://dx.doi.org/10.1139/z93-080

YAHNKE, C.J. 2006. Habitat use and natural history of small mammals in the central Paraguayan Chaco. Mastozool. Neotrop. 13(1):103-116.

Recebido em 29/06/2012

Versão reformulada recebida em 29/11/2012

Publicado em 14/01/2013 
